\section{Fixed-ratio performance for sucrose under nondeprived conditions following septal} lesions in rats ${ }^{1}$

GEORGE H. BUCKLAND ${ }^{2}$ and J. S. SCHWARTZBAUM, University of Rochester, Rochester, N.Y. 14627

Septal lesions were found to increase lever pressing for sucrose under nondeprived conditions with low and medium FR requirements of reinforcement up to FR-16. The effect did not persist at higher FRs but extended across different concentrations of sucrose at FR-8. The results provide additional evidence of a septal role in the inhibitory regulation of various incentive-reward processes.

Previous findings have suggested that components of the septal area normally exert inhibitory control over incentive-reward functions pertaining to gustatory and probably other classes of stimuli. Lesions in this region in rats increased consummatory response for a wide range of concentrations of sucrose solution during brief daily tests in a drinkometer apparatus (Beatty \& Schwartzbaum, 1968a), and they elevated daily fluid intake of a preferred saccharin solution (Beatty \& Schwartzbaum, 1967). The effects were not contingent upon food deprivation. Such lesions were also found to increase lever pressing for sucrose under FI contingencies (Beatty \& Schwartzbaum, $1968 b)$. Here the enhanced terminal rates of response during each FI appeared to reflect similar incentive-reward dysfunctions. The present investigation sought to extend this analysis of septal functions to an operant task that minimized temporal contingencies of reinforcement and, consequently, some form of suppression of response. Accordingly, performance for sucrose was studied under progressively increased fixed-ratio (FR) requirements. In addition, information was obtained on response to shifts in concentration of sucrose. Tests were conducted under nondeprived conditions of food and water.

$$
\text { METHOD }
$$

The Ss were 12 male Holtzman rats that

Fig. 1. Mean total FR responses per session (averaged across sessions) by septal and control groups at each $F R$ condition. Inset shows similar measures of performance at higher FRs following phase of experiment in which concentration of sucrose was varied. considered preliminary) were administered at FR-1, followed by three each at FR-2 and FR-4, and then by four sessions at FR-8; the initial response in each session was always reinforced. During the next phase, FR-8 was maintained and concentration of sucrose was varied systematically. The Ss were switched from $8 \%$ sucrose and received four sessions each with $2 \%, 32 \%$, and again $8 \%$ sucrose, in that order. They were then continued with $8 \%$ sucrose and tested for three session at each of FR-16, -32, and -64. Records of performance were obtained on counters for successive 5 -min periods of each session, as well as on cumulative recorders. However, responses made during operation of the dipper were segregated into a separate counter and did not contribute toward FR requirements.

\section{RESULTS}

It can be seen in Fig. 1 that septal lesions increased response output for $8 \%$ sucrose at all FRs from 1.8 and that the effect became progressively greater with the increase in FR requirements. Overall group differences and Group by FR interaction were each significant at the .05 level $(F=5.56$ and 3.02 , respectively, $\mathrm{df}=1 / 10$ and $3 / 30$ ). Only one of the six septal animals performed within the control range of values. ${ }^{3}$ These group differences in response output related basically to changes in patterns of postreinforcement pauses in lever pressing. The septal group tended to display less of a pause following each reinforcement and fewer sporadic long pauses in responding (Fig. 2). It exceeded the control group in ineffectual responding during resetting of the dipper by, e.g., averaging 76 additional responses per session at FR-1 and 117 at FR-8, compared to means of 35 and 39 ,

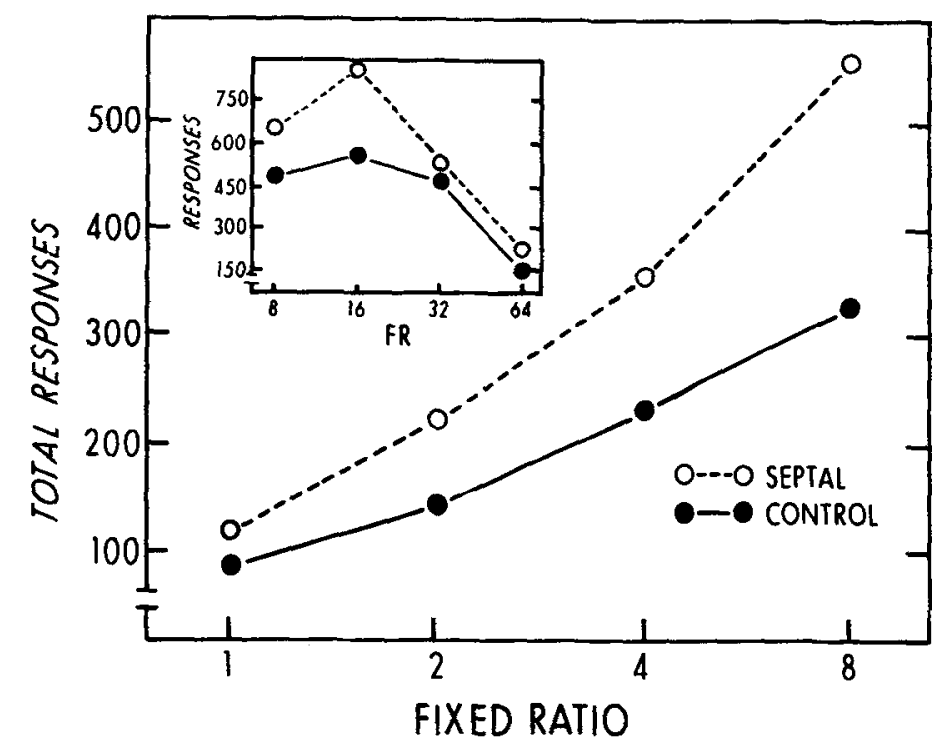

Psychon. Sci., 1970, Vol. 18 (5) 

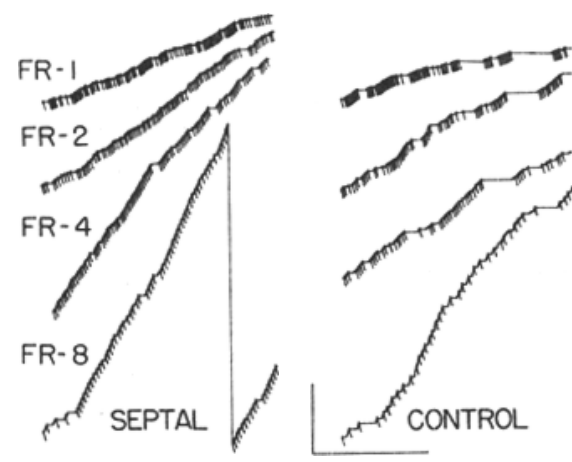

Fig. 2. Representative cumulative records of animals with median performance in each group at different FRs. Calibration lines indicate 120 responses (vertical) and $10 \mathrm{~min}$ (horizontal).

respectively, for the control group $(p<.05$ by $t$ test for each group difference and for the increase displayed by septal group). The septal animals, in this sense, showed less evidence of discriminating low FI contingencies $(2.0 \mathrm{sec})$ that were superimposed upon the FR. But, in general, they did not develop the pattern of postreinforcement pauses seen in control animals as FR requirements were increased to 4 and 8 . There appeared to be little group difference in time required to complete any sequence of FR responses once responding was initiated.

The inset of Fig. 1 summarizes performance for $8 \%$ sucrose at higher FRs following the phase of the experiment in which concentration of sucrose was varied. Group differences tended to persist at FR-8 and -16, although responding by septal animals during dipper-reset times decreased to control levels (38 additional responses per session). But of particular interest, responding by both groups declined at FR-32 and fell sharply at FR-64. The septal group showed no evidence of more persistent responding at these high FR values.

Finally, it may be seen in Fig. 3 that response output by both groups was highly sensitive to the properties of the reinforcer. The septal Ss displayed enhanced responding at all concentrations even though output varied systematically with concentration, and intrasession declines in response matched those of control Ss. The heightened response with $32 \%$ sucrose,

Fig. 3. Mean FR responses by septal and control groups during each half of session at different concentrations of sucrose. Data are based on last three sessions with each concentration. The order of presentation of the concentrations was $8 \%, 2 \%$, and $32 \%$. limited somewhat in septal Ss by ceiling effects, again related chiefly to reduction in postreinforcement pauses; septal and control groups now averaged 161 and 82 additional responses per session, respectively, during dipper-reset time, the highest values observed. Analysis of the concentration data, excluding the intrasession variable, confirmed overall group differences $(F=5.89, \quad d f=1 / 9$, $p<.05)$ and a concentration effect $(\mathrm{F}=21.62, \mathrm{df}=2 / 18, \mathrm{p}<.01)$.

In agreement with earlier observations (Beatty \& Schwartzbaum, 1967, 1968a), group differences in body weight occurred under the nondeprived conditions. The septal group gained $98 \mathrm{~g}$ during the 6 weeks following surgery, while the control group gained $151 \mathrm{~g}$ ( $\mathrm{p}<.05$ by U test). Nevertheless, the lighter control Ss, which approximated weights of the heavier septal Ss, still performed at a level below that of the latter animals.

\section{DISCUSSION}

Taken at face value, the present findings support the conclusion that septal lesions release or disinhibit incentive-reward processes associated with food stimuli having strong positive taste properties and that, as a consequence, these lesions induce more vigorous response for such rewarding stimuli under nondeprived conditions of food and water. Under the present conditions, this heightened response tendency was expressed in terms of output at low and medium FRs and not by extension of the FRs, which the animals were willing to tolerate in responding for sucrose. The results in general parallel earlier findings of consummatory behavior for sucrose (Beatty \& Schwartzbaum, 1968a) and are consistent with certain patterns of operant behavior displayed by septal animals under nondeprived conditions with FI contingencies (Beatty \& Schwartzbaum, 1968b).

However, the fact remains that group differences related basically to the proportion of time spent in lever-pressing as opposed to other activities. The differences in duration of postreinforcement pauses in responding at low and medium FRs largely accounted for group differences in response output. Therefore, one must consider the alternative possibilities: (1) that septal lesions somehow increased persistence of response apart from alterations in incentive-reward functions, or (2) that such lesions enhanced frustrative or aversive reactions to certain features of the test conditions which increased responding. The first alternative receives little support from the plasticity of response displayed by septal animals with respect to shifts in concentration of sucrose and from their failure to persist in responding at very high FRs. The second alternative, which receives support from the heightened aversive reactivity to quinine solutions and photic stimuli following septal lesions (Beatty \& Schwartzbaum, 1967; Green, Beatty, \& Schwartzbaum, 1967) and, especially, from more recent findings with DRL performance of septal animals following withholding of reinforcement (Caplan, 1968), is more difficult to evaluate in the present context. Fixed-ratio schedules of positive reinforcement may acquire aversive properties, especially at high ratio requirements (e.g., Thompson, 1964). While it is not unreasonable to suppose that such factors may have contributed to the failure of septal animals to sustain heightened responding at high FRs, their relevance at low and medium FR

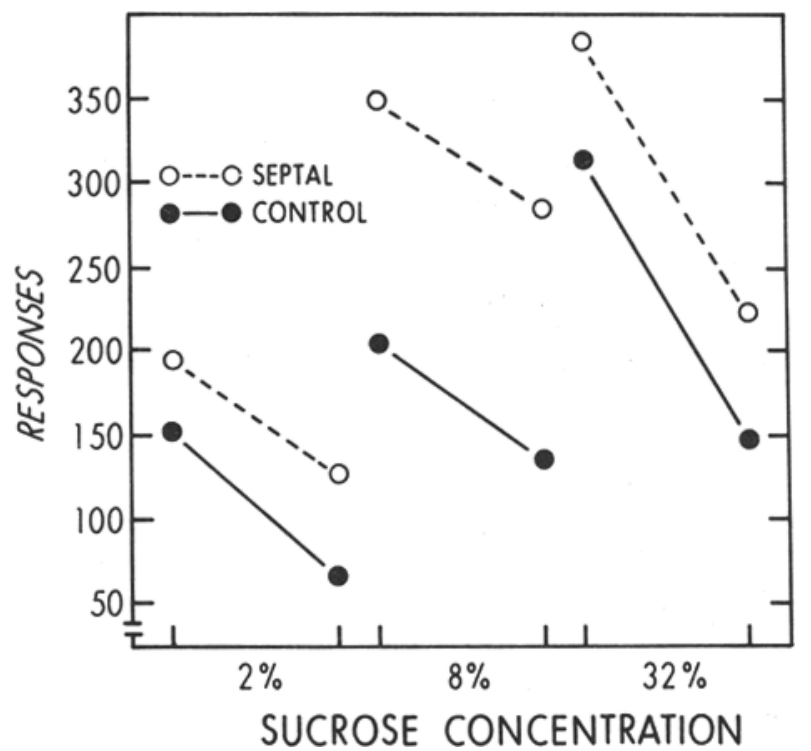


conditions remains unclear. The supposition that reduced postreinforcement pauses by septal Ss reflected enhanced frustrative or aversive reactions to $\mathrm{FR}$ or reinforcing conditions (e.g., withdrawal of sucrose during licking) receives questionable support from their increased responding during dipper-reset times in going from FR-1 to FR-8, since increased concentration of sucrose $(32 \%)$, which enhanced the reward properties of the solution, also served to elevate such responding in both groups. Indeed, pauses that normally develop as FR requirements increase may themselves reflect aversive consequences of the schedule (Ferster \& Skinner, 1957). In any event, it should be recognized that even if an aversive component were isolated as a factor, the knotty question would still remain as to whether enhanced aversive reactivity was primary or secondary to changes in positive incentive-reward functions. The latter still appear to represent major determinants of the observed behavioral alterations following septal lesions.

\section{REFERENCES}

BEATTY, W. W., \& SCHWARTZBAUM, J. S Enhanced reactivity to quinine and saccharin solutions following septal lesions in the rat. Psychonomic Science, 1967, 8, 483-484.

BEATTY, W. W., \& SCHWARTZBAUM, J. S. Consummatory behavior for sucrose following septal lesions in the rat. Joumal of Comparative \& Physiological Psychology, 1968a, 65, 93-102.

BEATTY, W. W., \& SCHWARTZBAUM, J. S. Commonality and specificity of behavioral dysfunctions following septal and hippocampal lesions in rats. Journal of Comparative \& Physiological Psychology, 1968b, 66, 60-68.

CAPLAN, M. The effects of lesions in the septal forebrain or the cingulate cortex on the timing behavior of rats. Unpublished doctoral dissertation, The City University of New York 1968.

FERSTER, C. B., \& SKINNER, B. F. Schedules of reinforcement. New York: Appleton-Century-Crofts, 1957

GREEN, R. H., BEATTY, W. W., \& SCHWARTZBAUM, J. S. Comparative effects of septo-hippocampal and caudate lesions on avoidance behavior in rats. Journal of Comparative \& Physiological Psychology, 1968, 66, 378-388.

HANSING, R. A., SCHWARTZBAUM, J. S., \& THOMPSON, J. B. Operant behavior following unilateral and bilateral caudate lesion in the rat. Journal of Comparative \& Physiological Psychology, 1968, 66, 378-388.

THOMPSON, D. M. Escape from $\mathrm{S}^{D}$ associated with fixed-ratio reinforcement. Journal of the Experimental Analysis of Behavior, 1964, 7, 1-8.

\section{NOTES}

1. Supported by Grant MH 14594-01 from NIMH.

2. Herbert Lehman predoctoral fellow.

3. This animal subsequently deviated to the extent of performing below all control Ss. Since it, therefore, questionably belonged to the same population as the other septal Ss, its subsequent data were excluded from group analysis. In effect, results with shifts in concentration and at high FRs are based on septal Ss that showed enhanced responding at low and medium FRs.

\section{Ambulatory behavior in the rat as a function of two methods of alcohol administration}

\section{ZALMAN AMIT and MURIEL H. STERN, McGill University, Montreal, P.Q., Canada}

When alcohol was administered directly into the stomach, ambulation scores of rats in an open-field apparatus were significantly higher than those following alcohol ingested by mouth, and higher following both methods of alcohol administration than after ingestion of water. This suggests that, while the pharmacologic properties of alcohol in general tend to reduce fear in the open-field situation, eliminating the aversive taste of the alcohol increases its fear-reducing effects.

In studies in which alcohol administration serves as an independent variable and a variety of behaviors as dependent variables, it is often assumed that only the volume and concentration of the alcohol solutions ingested are of significance. Recently several studies have suggested that, in addition to the pharmacological properties of alcohol solutions, the mode of alcohol administration is also an important variable affecting behavior. It seems that the mode of administration can either facilitate or inhibit alcohol's pharmacological effects on behavior.

Freed (1968) has reported that I.P. injections of alcohol are aversive and thus have punitive effects on rats in an approach-avoidance situation. A previous study by the present authors (Amit \& Stern, 1969) suggests that administration of alcohol via an intragastric fistula is not aversive, and that rats will work harder to receive intragastric loads of alcohol than they will to obtain alcohol orally in a lever-pressing situation.

The aim of the present study was to determine the effects in rats on open-field ambulatory behavior of two modes of alcohol administration.

\section{METHOD}

The Ss were 12 male hooded rats, 90 days old and weighing approximately $350 \mathrm{~g}$ at the beginning of the experiment. An intragastric fistula was implanted in each $S$ following the method suggested by Kohn (1951) and modified by Amit \& Stern (1969).

On the third day following surgery, using the techniques suggested by Cicero \& Myers (1968), a selection procedure was instituted to establish a low alcohol concentration for six animals for which the concentration established was $13 \%(\mathrm{w})$ and a high alcohol concentration for six animals for which the concentration was $18 \%$ (v). These concentrations remained constant throughout the experiment. Two experimental groups were then selected so that each contained three of the animals on low alcohol concentration and three of the animals on high alcohol concentration. Fourteen days after surgery, all animals were placed on a 22 -h $\mathrm{h}$ water-deprivation schedule.

On Test Day 1 and the 4 subsequent odd-numbered days, each animal in one of the groups received $7 \mathrm{ml}$ of alcohol solution infused directly into the stomach via a variable-speed infusion pump (Harvard Co., Dover, Mass.). On Test Day 2 and the 4 subsequent even-numbered days, the Ss in this group received the same quantity of alcohol by mouth from a Richter tube. The duration of the infusion, $8 \mathrm{~min}$, was equivalent to the mean duration of the oral intake of 22-h water-deprived animals determined in a pilot study. Ten minutes following the administration of alcohol, the animals were placed in the open-field apparatus.

The second group received saline solution (isotonic with the alcohol solutions) by infusion on the odd-numbered days and alcohol solution by infusion on the even-numbered days. All other procedures were the same as those applied to the first group.

The open-field apparatus used in testing 